\title{
Characterization of the Precision Manipulation Capabilities of Robot Hands via the Continuous Group of Displacements
}

\author{
Nicolas Rojas, Member, IEEE and Aaron M. Dollar, Senior Member, IEEE
}

\begin{abstract}
In robot hands, precision manipulation, defined as repositioning of a grasped object within the hand workspace without breaking or changing contact, is a fundamental operation for the accomplishment of highly dexterous manipulation tasks. This paper presents a method to characterize the precision manipulation capabilities of a given robot hand regardless of the particularities of the grasped object. The technique allows determining the composition of the displacement manifold (finite motion) of the grasped object relative to the palm of the robot hand and defining the displacements that can actually be controlled by the hand actuators without depending on external factors to the hand. The approach is based on a reduction of the graph of kinematic constraints related to the hand-object system through proper manipulations of the continuous subgroups of displacements generated by the hand joints and contacts. The proposed method is demonstrated through three detailed and constructive examples of common architectures of simplified multi-fingered hands.
\end{abstract}

\section{INTRODUCTION}

In the context of robot hands, dexterous manipulation can be broadly defined as the purposeful movement of an object within the hand by the relative movement of some fingers respect to the palm [1-3]. The importance of such kind of manipulation for the successful deployment of robots in realworld tasks is evident. However, the development of mechanical systems that reliably perform autonomous dexterous manipulations outside controlled environments is still an open problem [4]. While good progress is being made (e.g. [5-7]), much work remains to be done in both hand design and control schemes to implement dexterous manipulation movements.

In this work, we present a method to analyze the capabilities of robot hands for performing dexterous manipulation; the technique is useful for both of the described aspects for improving robot hands. In particular, we focus on manipulation activities in which a grasped object is repositioned within the hand without breaking or changing contact. These kinds of tasks are classified as within-hand prehensile manipulation with no motion at contact in the taxonomy of manipulation presented in [1]. For simplicity, we refer to this type of dexterous manipulation task as precision manipulation (Fig. 1), although certainly the concept has been used for a broader class of manipulations in the domain of robot hands [8, 9].

This work was supported in part by the National Science Foundation grants IIS-1317976 and IIS-0952856.

N. Rojas and A. M. Dollar are with the Department of Mechanical Engineering and Materials Science, Yale University, New Haven, CT USA. (e-mail: \{nicolas.rojas; aaron.dollar\}@yale.edu).

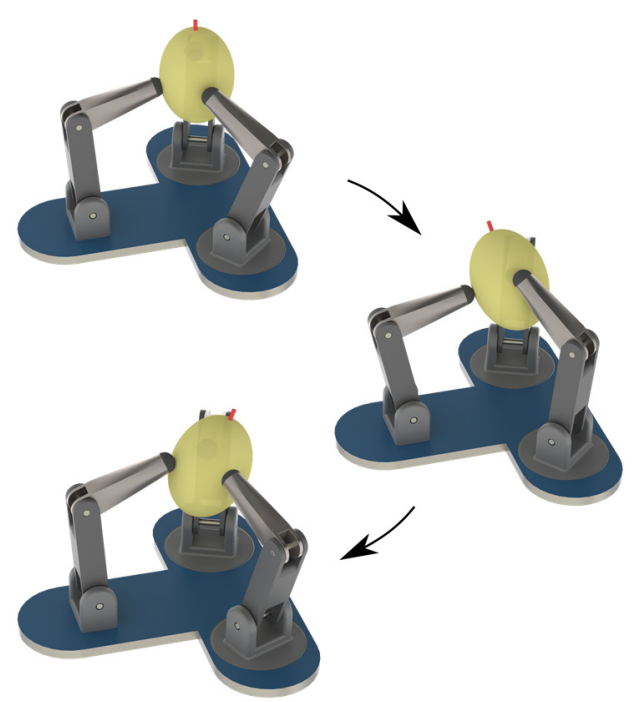

Fig. 1. During precision manipulation, a grasped object is repositioned within the hand workspace without breaking or changing contact. This task is classified in the hand-centric taxonomy of manipulation as within-hand prehensile manipulation with no motion at contact.

Examples of precision manipulation tasks include writing, inserting a key into a lock, and using scissors.

The aim of the proposed approach is to determine a mathematical characterization of the precision manipulation capabilities of a given robot hand. Such characterization is performed by determining the feasible movements to reposition a grasped object within the hand workspace without breaking or changing contact. This feasibility of motion refers to the composition of the displacement manifold (finite motion) of the object relative to the palm of the robot hand. A second related purpose is to define which of these possible displacements can actually be controlled by the hand actuators without depending on external factors to the hand. Our interest is in general displacement characteristics regardless the particularities of the grasped object -the instantaneous (or local) motion features and limitations resulting from, for instance, the particular dimensions of the hand-object system or the friction conditions are not considered here. This strategy of analysis is based on the Hervé's group-theoretic approach for the kinematics of mechanisms [10], a mathematical tool that has excelled in the type synthesis of parallel platforms [11-13]. Approaches based on screw theory could also be taken [14, 15]; but special attention should be paid to identifying the finite motion of the resulting instantaneous analysis, a step that could be difficult for some robot hand architectures.

This paper is organized as follows. Section II introduces the concept of the continuous group of displacements and its 
TABLE 1

SUBGROUPS OF DISPLACEMENTS

\begin{tabular}{|c|c|c|}
\hline Subgroup & $\begin{array}{c}\text { Kinematic } \\
\text { pair }\end{array}$ & Description \\
\hline$\{\mathbf{I}\}$ & & $\begin{array}{l}\text { The identity displacement. Rigid } \\
\text { connection between bodies, no } \\
\text { relative motion ( } 0 \text { degrees of } \\
\text { freedom })\end{array}$ \\
\hline$\{\mathbf{R}(N, \boldsymbol{u})\}$ & $\begin{array}{l}\text { Revolute } \\
\text { joint }\end{array}$ & $\begin{array}{l}\text { Rotation about the axis determined } \\
\text { by the unit vector } \boldsymbol{u} \text { and point } N \text { ( } 1 \\
\text { degree of freedom) }\end{array}$ \\
\hline$\{\mathbf{T}(\boldsymbol{v})\}$ & $\begin{array}{c}\text { Prismatic } \\
\text { joint }\end{array}$ & $\begin{array}{l}\text { Translation parallel to the unit vector } \\
\boldsymbol{v} \text { (1 degree of freedom) }\end{array}$ \\
\hline$\{\mathbf{G}(\boldsymbol{v})\}$ & Planar joint & $\begin{array}{l}\text { Planar gliding motion determined by } \\
\text { the unit normal vector } \boldsymbol{v} \text { ( } 3 \text { degrees } \\
\text { of freedom) }\end{array}$ \\
\hline$\{\mathbf{S}(N)\}$ & $\begin{array}{c}\text { Spherical } \\
\text { joint }\end{array}$ & $\begin{array}{l}\text { Spherical rotation about a point } N(3 \\
\text { degrees of freedom) }\end{array}$ \\
\hline
\end{tabular}

subgroups. Section III discusses kinematic constraints as subsets of the continuous group of displacements, showing the operations to properly reduce a type of graph of kinematic constraints for computing the possible relative motion between some links of its associated kinematic chain. In section IV, the introduced ideas are demonstrated through three detailed and constructive examples of common architectures of simplified multi-fingered hands, with the purpose of highlighting its potential and enlightening its use in other finger/palm layouts. Finally, we conclude and present directions for future work in section V.

\section{The Continuous Group of Displacements}

A group is basically a set combined with an operation, usually called multiplication or group operation, in which its elements satisfy four fundamental properties, namely: closure, associativity, the existence of identity, and the existence of inverses. A subgroup is a subset of a group that is itself a group under the same operation. In the context of robotics, a relevant example of group is the set of all the isometries of $\mathbb{R}^{3}$ (the three dimensional Euclidean space) that maintain handedness -i.e. the transformations that preserve a metric, excluding reflections- combined with the composition operation, denoted herein by the interpunct symbol $(\cdot)$. This group is usually called the proper Euclidean group [16].

It is known that such group of rigid motions is also a Lie group, that is, a continuous group of transformations [10, 17]. Henceforth, we denote this group as the continuous group of displacements, $\{\mathbf{D}\}$, that corresponds to a 6dimensional manifold, or 6-manifold. Hence, six parameters, or degrees of freedom, are required to define the location of a free body in space. In general, any motion of a rigid body can be described by a subset of $\{\mathbf{D}\}$, which may be either a subgroup or just a submanifold. It is important to clarify that, in any Cartesian reference frame, the composition of rigid motions (proper isometries) can be represented by a matrix product; the corresponding continuous group (Lie group) of matrices is normally denoted as $\mathrm{SE}(3)$, the special Euclidean group acting on $\mathbb{R}^{3}[18,19]$.

In this work, we build from the ideas of Hervé [10], who was, to the authors' knowledge, the first in proposing a group-theoretic approach for the kinematics of mechanisms and robotic systems. In his seminal paper, Hervé presented a detailed analysis of the subgroups of $\{\mathbf{D}\}$. An example of these subgroups is $\{\mathbf{R}(N, \boldsymbol{u})\}$, the subgroup of rotations around a fixed axis $(N, \boldsymbol{u})$, that is defined by a unit vector $\boldsymbol{u}$, parallel to the axis, and a point $N$ that belongs to it. The significance for robotics of these concepts lies in the fact that the rigid connection between two bodies and the relative motion allowed by any lower kinematic pair constitute subgroups of the continuous group of displacements. Table I presents a description of some of the subgroups of $\{\mathbf{D}\}$, with their associated lower kinematic pair, that are relevant for our discussion. For a complete list of subgroups, the interested reader is addressed to [20].

From the subgroups of the continuous group of displacements presented in Table I, $\{\mathbf{R}(N, \boldsymbol{u})\}$ and $\{\mathbf{T}(\boldsymbol{v})\}$ are the more fundamental because through their composition the other subgroups are formed. In this way, by a proper use of the group properties, these subgroups $(\{\mathbf{G}(\boldsymbol{v})\}$ and $\{\mathbf{S}(N)\})$ can be written in different equivalent ways. For instance, $\{\mathbf{G}(\boldsymbol{v})\}$, the subgroup of planar gliding motions is generated from the composition of $\{\mathbf{T}(\boldsymbol{v})\},\{\mathbf{T}(\boldsymbol{w})\}$, and $\{\mathbf{R}(N, \boldsymbol{u})\}$, provided $\boldsymbol{u}$ is perpendicular $(\perp)$ to the plane formed by vectors $\boldsymbol{v}$ and $\boldsymbol{w}$. That is, $\{\mathbf{G}(\boldsymbol{v})\}=\{\mathbf{T}(\boldsymbol{v})\}$. $\{\mathbf{T}(\boldsymbol{w})\} \cdot\{\mathbf{R}(N, \boldsymbol{u})\} . \quad$ Since $\quad\{\mathbf{R}(N, \boldsymbol{u})\} \cdot\{\mathbf{R}(N, \boldsymbol{u})\}=$ $\{\mathbf{R}(N, \boldsymbol{u})\} \quad(\forall x, x \in\{\mathbf{R}(N, \boldsymbol{u})\} \cdot\{\mathbf{R}(N, \boldsymbol{u})\}, x \in\{\mathbf{R}(N, \boldsymbol{u})\})$ and $\{\mathbf{T}(\boldsymbol{w})\} \cdot\{\mathbf{R}(N, \boldsymbol{u})\}=\{\mathbf{R}(O, \boldsymbol{u})\}$, with $\boldsymbol{u} \perp \boldsymbol{w}$ and $O \neq$ $N$, we have

$$
\begin{aligned}
\{\mathbf{G}(\boldsymbol{v})\} & =\{\mathbf{T}(\boldsymbol{v})\} \cdot\{\mathbf{T}(\boldsymbol{w})\} \cdot\{\mathbf{R}(N, \boldsymbol{u})\} \\
& =\{\mathbf{T}(\boldsymbol{v})\} \cdot\{\mathbf{T}(\boldsymbol{w})\} \cdot\{\mathbf{R}(N, \boldsymbol{u})\} \cdot\{\mathbf{R}(N, \boldsymbol{u})\} \\
& =\{\mathbf{T}(\boldsymbol{v})\} \cdot\{\mathbf{R}(O, \boldsymbol{u})\} \cdot\{\mathbf{R}(N, \boldsymbol{u})\} \\
& =\{\mathbf{R}(P, \boldsymbol{u})\} \cdot\{\mathbf{R}(O, \boldsymbol{u})\} \cdot\{\mathbf{R}(N, \boldsymbol{u})\} .
\end{aligned}
$$

Other representations of the subgroup $\{\mathbf{G}(\boldsymbol{v})\}$ can be derived following an equivalent procedure. For the case of the subgroup $\{\mathbf{S}(N)\}$, using the property of closure, it can be straightforwardly proven that $\{\mathbf{S}(N)\}=\{\mathbf{R}(N, \boldsymbol{i})\}$. $\{\mathbf{R}(N, \boldsymbol{j})\} \cdot\{\mathbf{R}(N, \boldsymbol{k})\}$ provided that $\boldsymbol{i}, \boldsymbol{j}$, and $\boldsymbol{k}$ are linearly independent vectors [19].

\section{GRAPHS OF KINEMATIC CONSTRAintS}

Given two bodies $m$ and $n$ in a Euclidean space, a kinematic constraint can be defined as the subset of the continuous group of displacements associated to the allowed motion of the body $m$ relative to body $n$. Thus, for example, the kinematic constraint of a free body in $\mathbb{R}^{3}$ is $\{\mathbf{D}\}$ and that of a cube constrained to move on a planar surface of normal $\boldsymbol{u}$ is $\{\mathbf{G}(\boldsymbol{u})\}$. If there exists a third body $o$ with a constrained motion relative to the bodies $m$ and $n$, we can construct a directed graph of three nodes and three directed edges that describe the restrictions of motion in the system. The nodes correspond to the bodies and the edges to the kinematic constraints between them. Such graph is called a graph of kinematic constraints [21, 22]. 
(a)

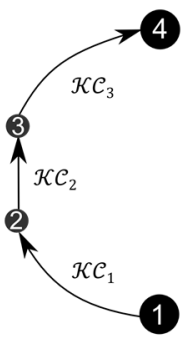

(b)
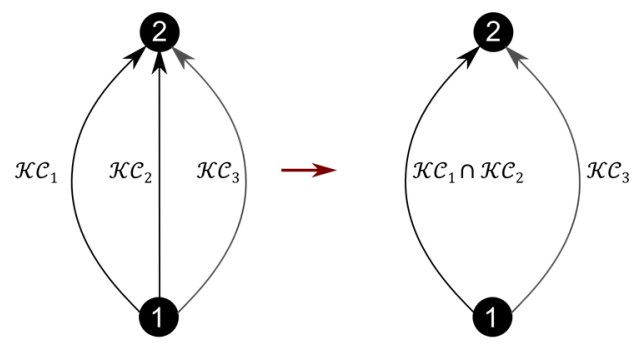

Fig. 2. Basic operations of reduction in graphs of kinematic constraints: serial reduction (a) and parallel reduction (b).

A mechanism, or kinematic chain, is a system of rigid bodies (links) interconnected by kinematic pairs (joints). A kinematic pair is basically a connection between links that constraints their relative motions -in this work we only consider lower pairs. Thus, a kinematic chain of $\mathrm{L}$ links and J joints can be naturally represented as a graph of kinematic constraints of $\mathrm{L}$ nodes and $\mathrm{J}$ edges. The fundamental geometric problem in a kinematic chain is to determine the possible relative motion of a link $\mathrm{i}$ respect to a linkj. In terms of a graph of kinematic constraints, this problem consists in finding a reduction of two nodes and one edge of the original graph representing the kinematic chain, that is, finding a single equivalent kinematic constraint between the nodes associated to links $\mathrm{i}$ and $\mathrm{j}$.

In order to reduce a graph of kinematic constraints for obtaining the equivalent constraint between two nodes, two operations can be defined [10], namely: i) serial reduction and ii) parallel reduction. The operation of serial reduction can be applied to a set of nodes that are in series. The reduction is performed by computing the composition of the kinematic constraints involved in the nodes. Thus, if the series has $\mathrm{N}$ nodes ( $\mathrm{N}-1$ edges), we get two nodes and a single edge after the reduction [Fig. 2(a)]. The operation of parallel reduction can be applied to any two nodes that are connected by at least two edges. The reduction is performed by computing the intersection of the kinematic constraints associated to two selected edges. If the two involved nodes have E edges, after the reduction we get two nodes and E -1 edges [Fig. 2(b)].

The intersection of two kinematic constraints, that is, two subsets of the continuous group of displacements is basically the intersection as in set theory [19], taking into account that the intersection of some subgroups of $\{\mathbf{D}\}$ generates a subgroup besides the identity displacement. For instance, the intersection $(\Omega)$ between two planar gliding motions $\{\mathbf{G}(\boldsymbol{u})\}$ and $\{\mathbf{G}(\boldsymbol{v})\}$ is $\{\mathbf{T}(\boldsymbol{w})\}$, where $\boldsymbol{w}$ is a unit vector in the direction of the intersection of the two planes. Moreover, $\{\mathbf{G}(\boldsymbol{u})\} \cap\{\mathbf{S}(N)\}=\{\mathbf{R}(N, \boldsymbol{u})\} \quad$ and $\quad\{\mathbf{S}(O)\} \cap\{\mathbf{S}(P)\}=$ $\{\mathbf{R}(O, \widehat{\boldsymbol{o p}})\}=\{\mathbf{R}(P, \widehat{\boldsymbol{o p}})\}$ with $\widehat{\boldsymbol{o p}}=\overrightarrow{O P} /\|\overrightarrow{O P}\|$. The reader is advised that these operations can be deduced from the general intersection of the manifolds related to the subgroups - e.g. sphere-sphere intersection in the case of $\{\mathbf{S}(O)\}$ and $\{\mathbf{S}(P)\}$. A complete list of non-identity intersections between subgroups of displacements can be found in [14](Table C.3).

It has been showed that it is not always possible to reduce a graph of kinematic constraints to a single edge connecting two nodes by simply using serial and parallel reductions [22]. However, in the case of kinematic chains where two links are connected through one or more sub-kinematic chains with serially connected joints, it can be readily proven that this reduction always exists for such two links. This is the case of the kinematic chains resulting from the hand-object system in precision manipulation tasks of robot hands composed of serial fingers.

\section{Precision Manipulation Analysis}

In this section, we apply the introduced ideas about the continuous group of displacements and the operations of reduction in graphs of kinematic constraints to the precision manipulation analysis of robot hands. The objective of this study is, first, to define the feasible movements to reposition a grasped object within the hand workspace without breaking or changing contact, a task we refer to as "precision manipulation" and, second, to determine which of these possible displacements are controllable. By feasible movements we mean the displacement manifold (finite motion) of the object relative to the base or palm of the robot hand. Controllable movements refer to the subset of these feasible displacements that can actually be controlled by the hand actuators, as opposed to those that cannot, such as motion out of the plane of a two-fingered gripper.

This approach is based on a group-theoretic analysis of the kinematic constraints associated to the hand-object system. In this study, the contacts between the fingertips and the object are modeled as lower kinematic pairs, specifically, as spherical joints. Other more complex kinematic models of surface-surface contacts that are based on kinematic chains [23], and thus suitable for the presented method, are left for further research. We are interested in general displacement characteristics -the instantaneous (or local) motion features and limitations resulting from, for instance, the particular dimensions of the hand-object system or the friction conditions of the contacts, are not considered. The result of the proposed method is a mathematical characterization of the general within-hand manipulation capabilities of the hand regardless of the particularities of the grasped object.

In what follows, as examples of application of the proposed approach, we present the precision manipulation analysis of three architectures of simplified multi-fingered hands, namely, a 2-fingered hand with RR opposed fingers (2F-2RR), a 3-fingered hand with two RR opposed fingers and an opposable RR thumb (3F-3RR), and a 3-fingered hand with two UR fingers and an opposable RR thumb (3F- 


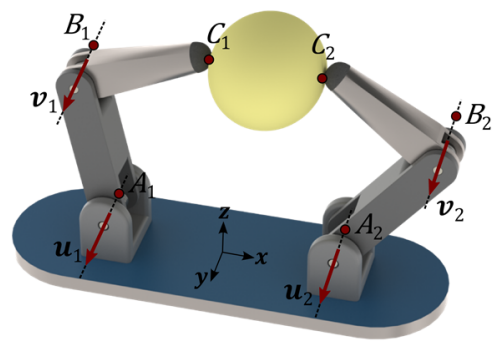

(a)

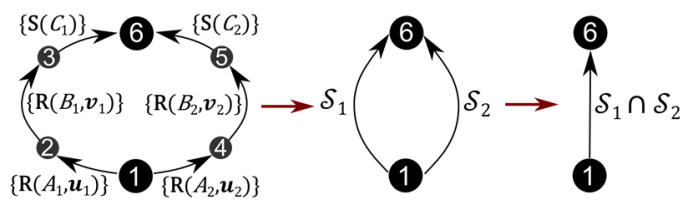

Fig. 3. Top: A 2F-2RR robot hand grasping an object with the notation used for the analysis of its within-hand prehensile manipulation capabilities (with no motion at contact). Bottom: The graph of kinematic constraints of the hand-object system for the $2 \mathrm{~F}-2 \mathrm{RR}$ hand

(a) and its corresponding reduction $(\mathbf{b}, \mathbf{c})$-see text for details.

2UR1RR). In this description, $\mathrm{R}$ and $\mathrm{U}$ stand for revolute pair and universal joint, respectively.

\section{A. Example 1: 2-Fingered Hand with Opposed Fingers $(2 F-2 R R)$}

Figure 3(top) shows a $2 \mathrm{~F}-2 \mathrm{RR}$ hand grasping, with its fingertips, a general object -represented as an egg-shaped body in the image. This hand is composed of two identical fingers with two links (proximal and distal) arranged in an opposed configuration. In each finger, the proximal link is connected to the palm through a revolute joint, which determines the motion plane of the finger. The proximal and distal links are connected by another revolute joint whose axis is parallel to the proximal one. The motion plane of both fingers is parallel. This finger/palm layout has been normally used for power grasping (e.g. [24]); but, despite its simple architecture, it has been shown that this hand can be used for complex manipulation operations such as picking up small objects from a flat surface (flip-and-pinch tasks) [25].

During tasks of precision manipulation, with the kinematic model of contact points as spherical pairs, the hand-object system of the $2 \mathrm{~F}-2 \mathrm{RR}$ hand is equivalent to a closed kinematic chain composed of six links with two revolute-revolute-spherical serial limbs that connect the palm of the robot hand, or base, to the grasped object. The mobility of such closed kinematic chain (6 links, 6 joints in $\mathbb{R}^{3}$ with a total number of 10 degrees of freedom in the joints) is, applying the Hunt's form of the ChebychevGrübler-Kutzbach criterion [26], 4. This implies that the feasible movements of a grasped object respect to the base correspond to a 4-manifold (embedded in $\mathbb{R}^{3}$ ), in other words, the object has 4 degrees of freedom. The questions are: how is the composition of the displacement related to these degrees of freedom? What are the involved axes in such motion? And, can all these movements be controlled by the hand actuators? Next, we present how to apply the continuous group of displacements method to resolve these relevant questions.

According to the notation of Fig. 3(top), for the left finger, the axis of the ground revolute joint (or proximal joint) is determined by a unit vector $\boldsymbol{u}_{1}$, that is parallel to the $y$-axis of the palm's reference frame $\left(\boldsymbol{u}_{1} \| \boldsymbol{y}\right)$, and any point, say $A_{1}$, that belongs to the line defined by the rotational axis. This kinematic pair corresponds to a kinematic constraint that forms the subgroup of displacements $\left\{\mathbf{R}\left(A_{1}, \boldsymbol{u}_{1}\right)\right\}=$ $\left\{\mathbf{R}\left(A_{1}, \boldsymbol{y}\right)\right\}$ that restrict the movement between the proximal link and the palm. Similarly, for the case of the distal joint, the generated subgroup is $\left\{\mathbf{R}\left(B_{1}, \boldsymbol{v}_{1}\right)\right\}=\left\{\mathbf{R}\left(B_{1}, \boldsymbol{y}\right)\right\}$. Finally, assuming that the contact point between the fingertip and the object is $C_{1}$, the motion constraint between the two bodies generates the subgroup $\left\{\mathbf{S}\left(C_{1}\right)\right\}$, that results from the spherical joint model of contacts. By replicating this analysis in the right finger, we can construct the graph of kinematic constraints of this hand-object system as depicted in Fig. 3 (bottom[a]).

In order to obtain a mathematical characterization of the displacement manifold of the grasped object relative to the palm of the 2F-2RR hand, we employ the operations of reduction, introduced in section III, to the corresponding graph of kinematic constraints. First, using the notation of Fig. 3(bottom[a]), we apply serial reduction to the nodes 1 , 2,3 , and 6 . Then, we get,

$$
\mathcal{S}_{1}=\left\{\mathbf{R}\left(A_{1}, \boldsymbol{y}\right)\right\} \cdot\left\{\mathbf{R}\left(B_{1}, \boldsymbol{y}\right)\right\} \cdot\left\{\mathbf{S}\left(C_{1}\right)\right\},
$$

where $\mathcal{S}_{1}$ is a kinematic constraint defined as the subset of the group of rigid-body displacements resulting from the composition operation of the subgroups involved in the related nodes. Now, since the subgroup $\left\{\mathbf{R}\left(C_{1}, \boldsymbol{y}\right)\right\}$ is a proper subset of the subgroup $\left\{\mathbf{S}\left(C_{1}\right)\right\}$, that is, $\left\{\mathbf{R}\left(C_{1}, \boldsymbol{y}\right)\right\} \subset$ $\left\{\mathbf{S}\left(C_{1}\right)\right\}$, then, by the property of closure, we get $\left\{\mathbf{R}\left(C_{1}, \boldsymbol{y}\right)\right\} \cdot$ $\left\{\mathbf{S}\left(C_{1}\right)\right\}=\left\{\mathbf{S}\left(C_{1}\right)\right\} \quad\left(\forall x, x \in\left\{\mathbf{R}\left(C_{1}, \boldsymbol{y}\right)\right\} \cdot\left\{\mathbf{S}\left(C_{1}\right)\right\}\right.$, $\left.x \in\left\{\mathbf{S}\left(C_{1}\right)\right\}\right)$. Hence,

$$
\begin{aligned}
\mathcal{S}_{1} & =\left\{\mathbf{R}\left(A_{1}, \boldsymbol{y}\right)\right\} \cdot\left\{\mathbf{R}\left(B_{1}, \boldsymbol{y}\right)\right\} \cdot\left\{\mathbf{R}\left(C_{1}, \boldsymbol{y}\right)\right\} \cdot\left\{\mathbf{S}\left(C_{1}\right)\right\} \\
& =\{\mathbf{G}(\boldsymbol{y})\} \cdot\left\{\mathbf{S}\left(C_{1}\right)\right\} .
\end{aligned}
$$

Note that the kinematic constraint $\mathcal{S}_{1}$ is a subset, but not a subgroup, of $\{\mathbf{D}\}$. Such subset corresponds to a 6-manifold.

Applying the same serial reduction to the nodes 1, 4, 5, and 6, we get (with $\boldsymbol{u}_{2}\left\|\boldsymbol{v}_{2}\right\| \boldsymbol{y}$ )

$$
\mathcal{S}_{2}=\{\mathbf{G}(\boldsymbol{y})\} \cdot\left\{\mathbf{S}\left(C_{2}\right)\right\} .
$$

Thus, after the application of the two serial reductions to the original graph of kinematic constraints, a reduced graph of two nodes with two edges is obtained, see Fig. 3(bottom[b]). The nodes of such graph are the base, the palm of the robot hand, and the grasped object, both connected by the kinematic constraints $\mathcal{S}_{1}$ and $\mathcal{S}_{2}$.

The final operation in the graph of kinematic constraints to get the subset of displacements of the grasped object, that is, to reduce the graph to two nodes with a single edge, is a parallel reduction applied to the kinematic constraints $\mathcal{S}_{1}$ and $\mathcal{S}_{2}$ [Fig. 3(bottom[c])]. Then, we have

$$
\begin{aligned}
\mathcal{P}_{1} & =\mathcal{S}_{1} \cap \mathcal{S}_{2} \\
& =\{\mathbf{G}(\boldsymbol{y})\} \cdot\left\{\mathbf{S}\left(C_{1}\right)\right\} \cap\{\mathbf{G}(\boldsymbol{y})\} \cdot\left\{\mathbf{S}\left(C_{2}\right)\right\} \\
& =\{\mathbf{G}(\boldsymbol{y})\} \cdot\left(\left\{\mathbf{S}\left(C_{1}\right)\right\} \cap\left\{\mathbf{S}\left(C_{2}\right)\right\}\right)
\end{aligned}
$$




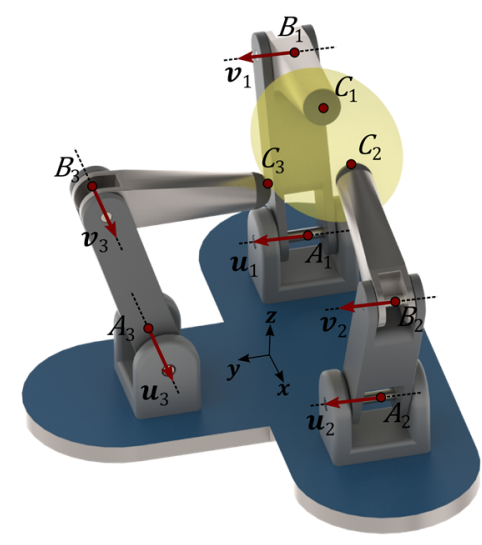

(a)

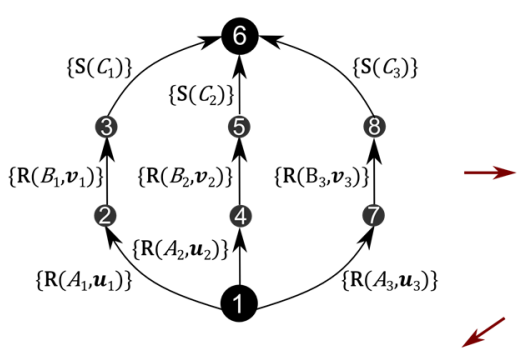

(c)

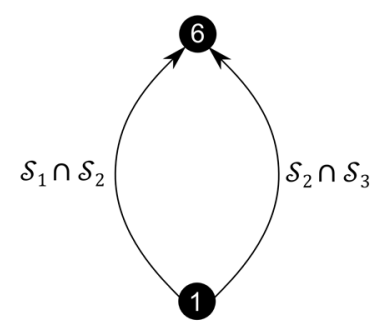

(d)

,

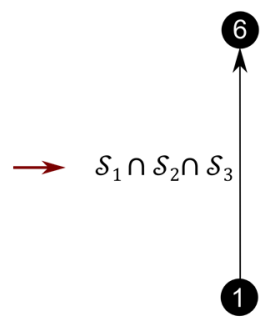

Fig. 4. Top: A 3F-3RR robot hand grasping an object with the notation used for the analysis of its precision manipulation capabilities. Bottom: The graph of kinematic constraints of the hand-object system for the 3F-3RR hand (a) and its corresponding reduction $(\mathbf{b}, \mathbf{c}, \mathbf{d})-$ see text for details.

$$
=\{\mathbf{G}(\boldsymbol{y})\} \cdot\left\{\mathbf{R}\left(C_{1}, \widehat{\boldsymbol{c}_{1} \boldsymbol{c}_{2}}\right)\right\} .
$$

The above equation implies that the feasible movements of a grasped object with a $2 \mathrm{~F}-2 \mathrm{RR}$ hand are the composition of a planar gliding displacement parallel to the $x Z$-plane (two translations and one rotation about the normal to the plane) and a rotation about the axis defined by the contact points $C_{1}$ and $C_{2}$. Note that, as required, the obtained finite displacement is a 4-manifold.

For determining if the four degrees of freedom of the grasped object can be controlled by the hand actuators, we just have to lock the input joints in the above analysis, that is, to equal all the controlled joints to $\{\mathbf{I}\}$, the identity displacement. If the resulting finite displacement from this process is the identity $\{\mathbf{I}\}$, then the actuators can control the different degrees of freedom because of the system becomes rigid. In a $2 \mathrm{~F}-2 \mathrm{RR}$ hand usually all four joints are actuated i.e. $\left\{\mathbf{R}\left(A_{1}, \boldsymbol{y}\right)\right\}=\left\{\mathbf{R}\left(A_{2}, \boldsymbol{y}\right)\right\}=\left\{\mathbf{R}\left(B_{1}, \boldsymbol{y}\right)\right\}=\left\{\mathbf{R}\left(B_{2}, \boldsymbol{y}\right)\right\}=$ $\{\mathbf{I}\}$ in the previous analysis. It can be verified that, in such a case, the obtained subset of $\{\mathbf{D}\}$ following the explained procedure is $\left\{\mathbf{R}\left(C_{1}, \widehat{\boldsymbol{c}_{1} \boldsymbol{c}_{2}}\right)\right\}$. In consequence, only 3 of the 4 degrees of freedom of the grasped object are controllable. The rotation about the axis defined by the contact points $C_{1}$ and $C_{2}$ cannot be controlled by the actuators and depends on other external factors, such as contact friction and mass/disturbance forces.

\section{B. Example 2: 3-Fingered Hand with Opposed Fingers and Opposable Thumb (3F-3RR)}

Figure 4(top) shows a 3-fingered hand with opposed RR fingers and an opposable RR thumb grasping, with its fingertips, a general object. This hand, called herein the $3 \mathrm{~F}-$ $3 R R$ hand, corresponds to a $2 F-2 R R$ hand, the layout studied in the last section, with an additional opposed finger. The motion plane of this last element is perpendicular to those defined by the first couple of fingers. This hand layout is useful for manipulating small objects or grasping elements that are placed in difficult positions [27]. The architecture is an alternative to the more popular arrangement of two fingers with parallel motion planes and one opposable thumb [28-30]. Both designs are in fact particular configurations of the more general 3-fingered hand with two UR fingers and an opposable RR thumb that is analyzed in the next subsection.

During tasks of precision manipulation, with the kinematic model of contact points as spherical pairs, the hand-object system of the 3F-3RR hand is equivalent to a closed kinematic chain composed of eight links with three revolute-revolute-spherical serial limbs that connect the base of the robot hand to the grasped object. The mobility of such closed kinematic chain ( 8 links, 9 joints in $\mathbb{R}^{3}$ with a total number of 15 degrees of freedom in the joints) is 3 . This implies that the feasible movements of a grasped object respect to the base correspond to a 3-manifold (embedded in $\mathbb{R}^{3}$ ), then, the object has 3 degrees of freedom. Next, similar to the analysis carried out for the $2 \mathrm{~F}-2 \mathrm{RR}$ hand, we present a mathematical characterization of the precision manipulation capabilities of the 3F-3RR hand using the method of the continuous group of displacements.

According to the notation of Fig. 4(top), let us call finger 1 , finger 2 , and finger 3 , the fingers with contact points $C_{1}$, $C_{2}$, and $C_{3}$, correspondingly. Fingers 1 and 2 have the same configuration (and notation) than the $2 \mathrm{~F}-2 \mathrm{RR}$ hand. In the case of finger 3 , the axis of the ground revolute joint, that is defined by the unit vector $\boldsymbol{u}_{3}$ and the point $A_{3}$, is parallel to the $x$-axis. Recall that, in this hand, the axes of the revolute distal joints are parallel to the axes of the proximal joints. The resulting graph of kinematic constraints for the handobject system in a 3F-3RR hand is depicted in Fig. 4(bottom[a]). This graph is composed of eight nodes and nine edges, related to number of links and joints of the associated kinematic chain, respectively.

For obtaining the mathematical characterization of the displacement manifold of a grasped object relative to the palm of a 3F-3RR hand, that is, to reduce the graph to a graph of two nodes with a single kinematic constraint, we firstly apply the operation of serial reduction to the sets of nodes $\{1,2,3,6\},\{1,4,5,6\}$, and $\{1,6,7,8\}$. From equations (3) and (4), we know that such operation for the sets 
$\{1,2,3,6\}$ and $\{1,4,5,6\}$ yields $\mathcal{S}_{1}=\{\mathbf{G}(\boldsymbol{y})\} \cdot\left\{\mathbf{S}\left(C_{1}\right)\right\}$ and $\mathcal{S}_{2}=\{\mathbf{G}(\boldsymbol{y})\} \cdot\left\{\mathbf{S}\left(C_{2}\right)\right\}$, respectively. Similarly, for the case of the set of nodes $\{1,6,7,8\}$, we get (with $\boldsymbol{u}_{3}\left\|\boldsymbol{v}_{3}\right\| \boldsymbol{x}$ )

$$
\begin{aligned}
\mathcal{S}_{3} & =\left\{\mathbf{R}\left(A_{3}, \boldsymbol{u}_{3}\right)\right\} \cdot\left\{\mathbf{R}\left(B_{3}, \boldsymbol{v}_{3}\right)\right\} \cdot\left\{\mathbf{S}\left(C_{3}\right)\right\} \\
& =\{\mathbf{G}(\boldsymbol{x})\} \cdot\left\{\mathbf{S}\left(C_{3}\right)\right\} .
\end{aligned}
$$

$\mathcal{S}_{1}, \mathcal{S}_{2}$, and $\mathcal{S}_{3}$ are kinematic constraints defined as subsets of the group of rigid-body displacements that result from the composition operation of the subgroups involved in their corresponding nodes. After these three serial operations, the original graph of kinematic constraints is reduced to a graph of two nodes with three edges [Fig. 4(bottom[b])].

In order to simplify the three kinematic constraints of the current reduced graph to a single couple of edges, we apply parallel reduction to, for instance, the kinematic constraints $\mathcal{S}_{1}$ and $\mathcal{S}_{2}$, and $\mathcal{S}_{2}$ and $\mathcal{S}_{3}$. We can choose in fact any possible combinations of edges - e.g. $\mathcal{S}_{1}$ and $\mathcal{S}_{2}$, and $\mathcal{S}_{1}$ and $\mathcal{S}_{3}$. From equation (5), it is known that $\mathcal{P}_{1}=\mathcal{S}_{1} \cap \mathcal{S}_{2}=$ $\{\mathbf{G}(\boldsymbol{y})\} \cdot\left\{\mathbf{R}\left(C_{1}, \widehat{\boldsymbol{c}_{1} \boldsymbol{c}_{2}}\right)\right\}$. Then, since the subgroup $\{\mathbf{G}(\boldsymbol{y})\}$ is generated from the composition of a translation $\{\mathbf{T}(\mathbf{z})\}$ and two rotations $\{\mathbf{R}(O, \boldsymbol{y})\}$ and $\{\mathbf{R}(W, \boldsymbol{y})\}$, where points $O$ and $W$ are any different points, for instance, $C_{3}$ and $C_{1}$, we have

$$
\begin{aligned}
\mathcal{P}_{1} & =\mathcal{S}_{1} \cap \mathcal{S}_{2} \\
& =\{\mathbf{G}(\boldsymbol{y})\} \cdot\left\{\mathbf{R}\left(C_{1}, \widehat{\boldsymbol{c}_{1} \boldsymbol{c}_{2}}\right)\right\} \\
& =\{\mathbf{T}(\mathbf{z})\} \cdot\left\{\mathbf{R}\left(C_{3}, \boldsymbol{y}\right)\right\} \cdot\left\{\mathbf{R}\left(C_{1}, \boldsymbol{y}\right)\right\} \cdot\left\{\mathbf{R}\left(C_{1}, \widehat{\boldsymbol{c}_{1} \boldsymbol{c}_{2}}\right)\right\} \\
& =\{\mathbf{T}(\mathbf{z})\} \cdot\left\{\mathbf{R}\left(C_{3}, \boldsymbol{y}\right)\right\} \cdot\left\{\mathbf{S}_{\mathbf{2}}\left(C_{1}\right)\right\},
\end{aligned}
$$

where $\left\{\mathbf{S}_{2}\left(C_{1}\right)\right\}$ is a submanifold included in $\left\{\mathbf{S}\left(C_{1}\right)\right\}$ and defined as the composition of two different subgroups of rotations whose axes meet at the point $C_{1}[31,32]$, provided the corresponding unit vectors are linearly independent, as it is the case of $\boldsymbol{y}$ and $\widehat{\boldsymbol{c}_{1} \boldsymbol{c}_{2}}$.

For the case of the kinematic constraints $\mathcal{S}_{2}$ and $\mathcal{S}_{3}$, we have

$$
\begin{aligned}
\mathcal{P}_{2} & =\mathcal{S}_{2} \cap \mathcal{S}_{3} \\
& =\{\mathbf{G}(\boldsymbol{y})\} \cdot\left\{\mathbf{S}\left(C_{2}\right)\right\} \cap\{\mathbf{G}(\boldsymbol{x})\} \cdot\left\{\mathbf{S}\left(C_{3}\right)\right\} \\
& =\{\mathbf{T}(\mathbf{z})\} \cdot\left\{\mathbf{R}\left(C_{3}, \boldsymbol{y}\right)\right\} \cdot\left\{\mathbf{R}\left(C_{2}, \boldsymbol{x}\right)\right\} \cdot\left\{\mathbf{R}\left(C_{2}, \widehat{\boldsymbol{c}_{2} \boldsymbol{c}_{3}}\right)\right\} \\
& =\{\mathbf{T}(\mathbf{z})\} \cdot\left\{\mathbf{R}\left(C_{3}, \boldsymbol{y}\right)\right\} \cdot\left\{\mathbf{S}_{\mathbf{2}}\left(C_{2}\right)\right\} .
\end{aligned}
$$

Recalling $\{\mathbf{G}(\boldsymbol{u})\} \cap\{\mathbf{G}(\boldsymbol{v})\}=\{\mathbf{T}(\boldsymbol{w})\}$ with $\boldsymbol{w}$ a unit vector in the direction of the intersection of the two planes, and $\{\mathbf{G}(\boldsymbol{u})\} \cap\{\mathbf{S}(N)\}=\{\mathbf{R}(N, \boldsymbol{u})\}$. Observe that equation (8) corresponds to a 4-manifold, as it is required by the closed kinematic chain associated to the kinematic constraints $\delta_{2}$ and $\delta_{3}$. After the application of the two presented parallel reductions, a graph of kinematic constraints of two nodes with two edges is obtained [Fig. 4(bottom[c])]. The nodes of such graph are the base of the robot hand and the grasped object, both connected by the kinematic constraints $\mathcal{P}_{1}=$ $\mathcal{S}_{1} \cap \mathcal{S}_{2}$ and $\mathcal{P}_{2}=\mathcal{S}_{2} \cap \mathcal{S}_{3}$.

The final operation in the graph of kinematic constraints to get the subset of displacements of the grasped object is a last parallel reduction applied to the constraints $\mathcal{P}_{1}$ and $\mathcal{P}_{2}$ [Fig. 4(bottom[d])]. Thus, we have

$$
\begin{aligned}
\mathcal{P}_{3}= & \mathcal{P}_{1} \cap \mathcal{P}_{2} \\
= & \{\mathbf{T}(\mathbf{z})\} \cdot\left\{\mathbf{R}\left(C_{3}, \boldsymbol{y}\right)\right\} \cdot\left\{\mathbf{S}_{2}\left(C_{1}\right)\right\} \cap\{\mathbf{T}(\mathbf{z})\} \\
& \left\{\mathbf{R}\left(C_{3}, \boldsymbol{y}\right)\right\} \cdot\left\{\mathbf{S}_{2}\left(C_{2}\right)\right\} \\
= & \{\mathbf{T}(\mathbf{z})\} \cdot\left\{\mathbf{R}\left(C_{3}, \boldsymbol{y}\right)\right\} \cdot\left(\left\{\mathbf{S}_{2}\left(C_{1}\right)\right\} \cap\left\{\mathbf{S}_{\mathbf{2}}\left(C_{2}\right)\right\}\right),
\end{aligned}
$$

since $\left\{\mathbf{R}\left(C_{1}, \widehat{\boldsymbol{c}_{1} \boldsymbol{c}_{2}}\right)\right\} \subset\left\{\mathbf{S}_{\mathbf{2}}\left(C_{1}\right)\right\},\left\{\mathbf{R}\left(C_{2}, \widehat{\boldsymbol{c}_{1} \boldsymbol{c}_{2}}\right)\right\} \subset\left\{\mathbf{S}_{\mathbf{2}}\left(C_{2}\right)\right\}$, and $\left\{\mathbf{R}\left(C_{1}, \widehat{\boldsymbol{c}_{1} \boldsymbol{c}_{2}}\right)\right\}=\left\{\mathbf{R}\left(C_{2}, \overline{\boldsymbol{c}_{1} \boldsymbol{c}_{2}}\right)\right\}$ (both points belong to the axis of rotation), $\left\{\mathbf{S}_{2}\left(C_{1}\right)\right\} \cap\left\{\mathbf{S}_{\mathbf{2}}\left(C_{2}\right)\right\}=\left\{\mathbf{R}\left(C_{1}, \widehat{\boldsymbol{c}_{1} \boldsymbol{c}_{2}}\right)\right\}$. Then, we finally get

$$
\mathcal{P}_{3}=\{\mathbf{T}(\mathbf{z})\} \cdot\left\{\mathbf{R}\left(C_{3}, \boldsymbol{y}\right)\right\} \cdot\left\{\mathbf{R}\left(C_{1}, \widehat{\boldsymbol{c}_{1} \boldsymbol{c}_{2}}\right)\right\} .
$$

Equation (10) implies that the feasible movements of a grasped object with a 3F-3RR hand are the composition of a translation along the $z$-axis, a rotation about the $y$-axis, and a rotation about the axis defined by the contact points $C_{1}$ and $C_{2}$. The obtained finite displacement is a 3-manifold, a result coherent with the mobility of the associated kinematic chain of the hand-object system.

As previously discussed in the analysis of the $2 \mathrm{~F}-2 \mathrm{RR}$ hand, for determining if the three degrees of freedom of the grasped object in a $3 \mathrm{~F}-3 \mathrm{RR}$ hand can be controlled by the hand actuators, we lock the input joints in the above displacement analysis to verify if the resulting motion is the identity $\{\mathbf{I}\}$. If we assume the fingers in the $3 \mathrm{~F}-3 \mathrm{RR}$ hand are fully actuated, it can be verified that the obtained subset of $\{$ D $\}$ is in fact such displacement. Actually, we can select any combination of three joints from the six available revolute joints in the hand to control the degrees of freedom of the grasped object.

\section{Example 3: 3-Fingered Hand with UR Fingers and Opposable RR Thumb (3F-2UR1RR)}

Our last example is a 3 -fingered hand with two UR fingers and an opposable RR thumb, called herein the 3F2UR1RR hand. This finger/palm layout is used in some popular commercial robot hands such as the Schunk Hand [33] or the Barrett Hand $^{1}[27,34]$ as well as in novel compliant underactuated hands recently presented [4]. Fig. 5(top) shows a representation of a 3F-2UR1RR hand grasping, with its fingertips, a general object. Next, as in the previous cases, we present a mathematical characterization of the precision manipulation capabilities of this hand using the continuous group of displacements method.

During tasks of precision manipulation, with the kinematic model of contact points as spherical pairs, the hand-object system of the 3F-2UR1RR hand is equivalent to a closed kinematic chain composed of eight links with two universal-revolute-spherical serial limbs and a revoluterevolute-spherical serial chain that connect the base of the robot hand to the grasped object. The mobility of this closed kinematic chain ( 8 links, 9 joints in $\mathbb{R}^{3}$ with a total number of 17 degrees of freedom in the joints) is 5 . Similar to the previous cases, this implies that the feasible movements of a grasped object respect to the base correspond to a 5manifold (embedded in $\mathbb{R}^{3}$ ), thus, the object has 5 degrees of freedom.

According to the notation of Fig. 5(top), let us call finger 1 , finger 2 , and finger 3 , the fingers with contact points $C_{1}$, $C_{2}$, and $C_{3}$, correspondingly. For the first finger, the

\footnotetext{
1 In the Ulrich's UPenn/Barrett Hand, the fingers are actually RRR kinematic chains with the axes of the two first joints perpendicular but not coincident (as it is the case in a universal joint). However, it can be proven that, from the kinematic viewpoint of our analysis, both topologies are equivalent.
} 


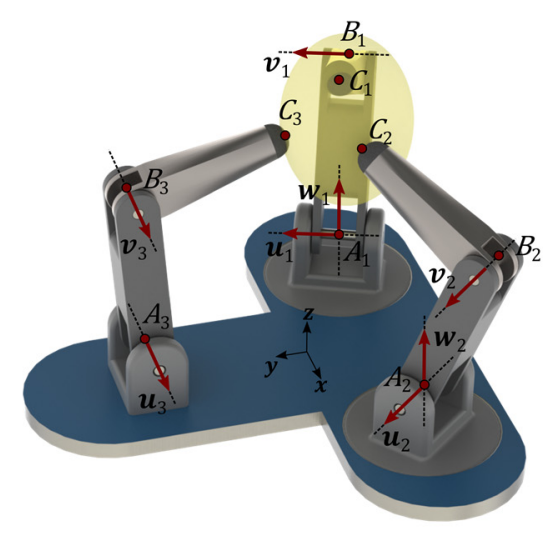

(a)

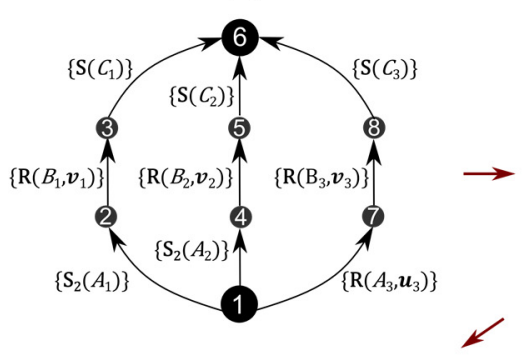

(c)

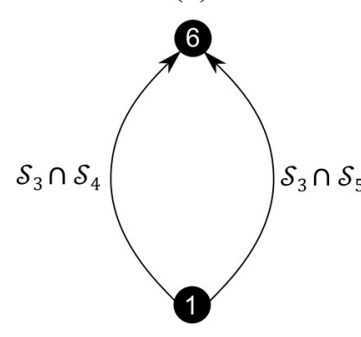

(d)

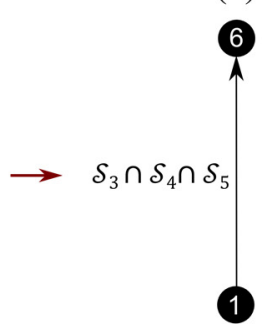

Fig. 5. Top: A 3F-2UR1R robot hand grasping an object with the notation used for its precision manipulation analysis. Bottom: The graph of kinematic constraints of the hand-object system for the 3F2UR1R hand (a) and its corresponding reduction (b, c, d) - see text for details.

proximal joint is a universal pair whose axes of rotation are determined by the unit vectors $\boldsymbol{u}_{1}$ and $\boldsymbol{w}_{1}$, that are parallel to the $x y$-plane and to the $z$-axis, respectively, and the meeting point of the axes, say $A_{1}$. This kinematic pair corresponds to a kinematic constraint that forms the submanifold $\left\{\mathbf{S}_{2}\left(A_{1}\right)\right\}=\left\{\mathbf{R}\left(A_{1}, \boldsymbol{z}\right)\right\} \cdot\left\{\mathbf{R}\left(A_{1}, \boldsymbol{u}_{1}\right)\right\}$, defined as the composition of two different subgroups of rotations whose axes meet at a single point. Taking into account that finger 1 and finger 2 have the same configuration, and that finger 3 is equivalent to the opposable thumb of the $3 \mathrm{~F}-3 \mathrm{RR}$ hand, then, for the hand-object system of the 3F-2UR1RR hand, we get the graph of kinematic constraints that is depicted in Fig. 5(bottom[a]).

In order to reduce the graph of kinematic constraints, we initially apply serial reductions as in the previous examples. Thus, for the case of nodes $1,2,3$, and 6 -related to the hand's first finger, we have (with $\boldsymbol{u}_{1} \| \boldsymbol{v}_{1}$ )

$$
\begin{aligned}
\mathcal{S}_{4}= & \left\{\mathbf{S}_{\mathbf{2}}\left(A_{1}\right)\right\} \cdot\left\{\mathbf{R}\left(B_{1}, \boldsymbol{v}_{1}\right)\right\} \cdot\left\{\mathbf{S}\left(C_{1}\right)\right\} \\
= & \left\{\mathbf{R}\left(A_{1}, \mathbf{z}\right)\right\} \cdot\left\{\mathbf{R}\left(A_{1}, \boldsymbol{u}_{1}\right)\right\} \cdot\left\{\mathbf{R}\left(B_{1}, \boldsymbol{u}_{1}\right)\right\} . \\
& \left\{\mathbf{R}\left(C_{1}, \boldsymbol{u}_{1}\right)\right\} \cdot\left\{\mathbf{S}\left(C_{1}\right)\right\}
\end{aligned}
$$

$$
=\left\{\mathbf{R}\left(A_{1}, \mathbf{z}\right)\right\} \cdot\left\{\mathbf{G}\left(\boldsymbol{u}_{1}\right)\right\} \cdot\left\{\mathbf{S}\left(C_{1}\right)\right\},
$$

where $\mathcal{S}_{4}$ is a kinematic constraint defined by the composition operation of the subgroups involved in the constraints of its corresponding nodes. Equation (11) is obtained following an expansion similar to that presented in equation (3). In the same way, for the case of nodes $1,4,5$, and 6 -related to finger 2 , we obtain

$$
\mathcal{S}_{5}=\left\{\mathbf{R}\left(A_{2}, \mathbf{z}\right)\right\} \cdot\left\{\mathbf{G}\left(\boldsymbol{u}_{2}\right)\right\} \cdot\left\{\mathbf{S}\left(C_{2}\right)\right\} .
$$

Finally, for the third finger (nodes 1, 6, 7, and 8), we have $\mathcal{S}_{3}=\{\mathbf{G}(\boldsymbol{x})\} \cdot\left\{\mathbf{S}\left(C_{3}\right)\right\}$ (see derivation of equations (3) and (6)).

After the above reductions we get a graph of kinematic constraints of two nodes and three edges [Fig. 5(bottom[b])]. To obtain a graph with a single couple of kinematic constraints, we apply parallel reduction to, for instance, the kinematic constraints $\mathcal{S}_{3}$ and $\mathcal{S}_{4}$, and $\mathcal{S}_{3}$ and $\mathcal{S}_{5}$. We can choose in fact any possible combinations of edges - e.g. $\mathcal{S}_{4}$ and $\mathcal{S}_{5}$, and $\mathcal{S}_{5}$ and $\mathcal{S}_{3}$. Then, for $\mathcal{S}_{3}$ and $\mathcal{S}_{4}$, we have

$$
\begin{aligned}
\mathcal{P}_{4} & =\mathcal{S}_{3} \cap \mathcal{S}_{4} \\
& =\{\mathbf{G}(\boldsymbol{x})\} \cdot\left\{\mathbf{S}\left(C_{3}\right)\right\} \cap \\
& \left\{\mathbf{R}\left(A_{1}, \mathbf{z}\right)\right\} \cdot\left\{\mathbf{G}\left(\boldsymbol{u}_{1}\right)\right\} \cdot\left\{\mathbf{S}\left(C_{1}\right)\right\} \\
& =\{\mathbf{T}(\mathbf{z})\} \cdot\left\{\mathbf{R}\left(C_{1}, \boldsymbol{x}\right)\right\} \cdot\left\{\mathbf{R}\left(C_{3}, \boldsymbol{u}_{1}\right)\right\} \cdot\left\{\mathbf{R}\left(C_{3}, \widehat{\boldsymbol{c}_{3} \boldsymbol{c}_{1}}\right)\right\} \\
& =\{\mathbf{T}(\mathbf{z})\} \cdot\left\{\mathbf{R}\left(C_{1}, \boldsymbol{x}\right)\right\} \cdot\left\{\mathbf{S}_{2}\left(C_{3}\right)\right\} \\
& =\{\mathbf{T}(\mathbf{z})\} \cdot\left\{\mathbf{R}\left(C_{1}, \boldsymbol{x}\right)\right\} \cdot\left\{\mathbf{R}\left(C_{3}, \boldsymbol{x}\right)\right\} \cdot\left\{\mathbf{S}_{2}\left(C_{3}\right)\right\} \\
& =\{\mathbf{G}(\boldsymbol{x})\} \cdot\left\{\mathbf{S}_{2}\left(C_{3}\right)\right\},
\end{aligned}
$$

where $\{\mathbf{G}(\boldsymbol{x})\} \cap\left\{\mathbf{G}\left(\boldsymbol{u}_{1}\right)\right\}=\{\mathbf{T}(\mathbf{z})\}, \quad\{\mathbf{G}(\boldsymbol{u})\} \cap\{\mathbf{S}(N)\}=$ $\{\mathbf{R}(N, \boldsymbol{u})\}$, and $\left\{\mathbf{S}_{\mathbf{2}}\left(C_{3}\right)\right\}=\left\{\mathbf{R}\left(C_{3}, \boldsymbol{x}\right)\right\} \cdot\left\{\mathbf{S}_{\mathbf{2}}\left(C_{3}\right)\right\}$. Note that $\left\{\mathbf{R}\left(A_{1}, \boldsymbol{z}\right)\right\}$ does not belong to any subgroup of $\mathcal{S}_{3} . \mathcal{P}_{4}$ is a 5manifold, as determined by the closed kinematic chain associated to the kinematic constraints $\mathcal{S}_{2}$ and $\mathcal{S}_{3}$. Analogously, for $\mathcal{S}_{3}$ and $\mathcal{S}_{5}$, we get

$$
\begin{aligned}
\mathcal{P}_{5} & =\mathcal{S}_{3} \cap \mathcal{S}_{5} \\
& =\{\mathbf{G}(\boldsymbol{x})\} \cdot\left\{\mathbf{S}\left(C_{3}\right)\right\} \cap \\
& \left\{\mathbf{R}\left(A_{2}, \mathbf{z}\right)\right\} \cdot\left\{\mathbf{G}\left(\boldsymbol{u}_{2}\right)\right\} \cdot\left\{\mathbf{S}\left(C_{2}\right)\right\} \\
& =\{\mathbf{T}(\mathbf{z})\} \cdot\left\{\mathbf{R}\left(C_{2}, \boldsymbol{x}\right)\right\} \cdot\left\{\mathbf{R}\left(C_{3}, \boldsymbol{u}_{2}\right)\right\} \cdot\left\{\mathbf{R}\left(C_{3}, \widehat{\boldsymbol{c}_{3} \boldsymbol{c}_{2}}\right)\right\} \\
& =\{\mathbf{T}(\mathbf{z})\} \cdot\left\{\mathbf{R}\left(C_{2}, \boldsymbol{x}\right)\right\} \cdot\left\{\mathbf{S}_{2}\left(C_{3}\right)\right\} \\
& =\{\mathbf{T}(\mathbf{z})\} \cdot\left\{\mathbf{R}\left(C_{2}, \boldsymbol{x}\right)\right\} \cdot\left\{\mathbf{R}\left(C_{3}, \boldsymbol{x}\right)\right\} \cdot\left\{\mathbf{S}_{\mathbf{2}}\left(C_{3}\right)\right\} \\
& =\{\mathbf{G}(\boldsymbol{x})\} \cdot\left\{\mathbf{S}_{2}\left(C_{3}\right)\right\} .
\end{aligned}
$$

After the application of the two above parallel reductions, a graph of kinematic constraints of two nodes with two edges is obtained [Fig. 5(bottom[c])].

For finally obtaining the subset of displacements of the grasped object, we apply a last parallel reduction to the constraints $\mathcal{P}_{4}$ and $\mathcal{P}_{5}$ [Fig. 5(bottom[d])]. Thus, we get

$$
\begin{aligned}
\mathcal{P}_{6} & =\mathcal{P}_{4} \cap \mathcal{P}_{5} \\
& =\{\mathbf{G}(\boldsymbol{x})\} \cdot\left\{\mathbf{S}_{2}\left(C_{3}\right)\right\} \cap\{\mathbf{G}(\boldsymbol{x})\} \cdot\left\{\mathbf{S}_{\mathbf{2}}\left(C_{3}\right)\right\} \\
& =\{\mathbf{G}(\boldsymbol{x})\} \cdot\left\{\mathbf{S}_{\mathbf{2}}\left(C_{3}\right)\right\} .
\end{aligned}
$$

The above equation implies that the feasible movements of a grasped object with a 3F-2UR1RR hand are the composition of a planar gliding displacement parallel to the $y z$-plane (two translations and one rotation about the normal to the plane) and two rotations about any two linearly independent axes that meet at point $C_{3}$, say, for instance, $\left(C_{3}, x\right)$ and $\left(C_{3}, \boldsymbol{y}\right)$. As necessary, the obtained finite displacement is a 5-manifold. Moreover, it can be verified that any selection of 
five actuated pairs in the hand from the possible eight joints (considering the universal joints as two revolute joints that can be independently actuated), generates the identity displacement. Hence, there are not uncontrollable degrees of freedom in the hand-object system.

\section{CONCLUSION}

We have presented a method, based on the continuous group of displacements and graphs of kinematic constraints, to characterize the precision manipulation capabilities of a robot hand. The approach is general and can be applied to any finger/palm layout or subset of it, provided the hand joints are lower kinematic pairs. The proposed technique can be used, for instance, in early stages of robot hand design to incorporate manipulation primitives needed to perform specific tasks. However, if a study of the topological properties of the resulting displacement manifolds is required, other techniques should be implemented. Several lines of future work can be identified within the scope of the proposed approach; we stand out: to extend the method to more complex contact models, as previously discussed, and to make automatic the analysis process.

\section{REFERENCES}

[1] I. M. Bullock, T. Feix, and A. M. Dollar, "Finding small, versatile sets of human grasps to span common objects," in IEEE International Conference on Robotics and Automation (ICRA), 2013, pp. 10681075.

[2] A. M. Okamura, N. Smaby, and M. R. Cutkosky, "An overview of dexterous manipulation," in IEEE International Conference on Robotics and Automation, 2000, pp. 255-262 vol.1.

[3] A. Bicchi, "Hands for dexterous manipulation and robust grasping: a difficult road toward simplicity," IEEE Transactions on Robotics and Automation, vol. 16, pp. 652-662, 2000.

[4] L. U. Odhner, L. P. Jentoft, M. R. Claffee, N. Corson, Y. Tenzer, R. R. Ma, M. Buehler, R. Kohout, R. D. Howe, and A. M. Dollar, "A Compliant, Underactuated Hand for Robust Manipulation," International Journal of Robotics Research, to appear.

[5] D. Aukes, S. Kim, P. Garcia, A. Edsinger, and M. R. Cutkosky, "Selectively compliant underactuated hand for mobile manipulation," in IEEE International Conference on Robotics and Automation (ICRA), 2012, pp. 2824-2829.

[6] M. Grebenstein, M. Chalon, W. Friedl, S. Haddadin, T. Wimböck, G. Hirzinger, and R. Siegwart, "The hand of the DLR Hand Arm System: Designed for interaction," The International Journal of Robotics Research, vol. 31, pp. 1531-1555, 2012.

[7] K. Yamaguchi, Y. Hirata, and K. Kosuge, "Development of robot hand with suction mechanism for robust and dexterous grasping," in IEEE/RSJ International Conference on Intelligent Robots and Systems (IROS), 2013, pp. 5500-5505.

[8] P. Michelman, "Precision object manipulation with a multifingered robot hand," IEEE Transactions on Robotics and Automation, vol. 14, pp. 105-113, 1998.

[9] T. Hasegawa, K. Murakami, and T. Matsuoka, "Grasp planning for precision manipulation by multifingered robotic hand," in IEEE International Conference on Systems, Man, and Cybernetics, 1999, pp. $762-767$ vol.6.

[10] J. M. Hervé, "Analyse structurelle des mécanismes par groupe des déplacements," Mechanism and Machine Theory, vol. 13, pp. 437450, 1978.

[11] J. Angeles, "The Qualitative Synthesis of Parallel Manipulators," Journal of Mechanical Design, vol. 126, pp. 617-624, 2004.

[12] L. Qinchuan and J. M. Hervé, "1T2R Parallel Mechanisms Without Parasitic Motion," IEEE Transactions on Robotics, vol. 26, pp. 401410, 2010.
[13] C. Fan, H. Liu, and Y. Zhang, "Type synthesis of 2T2R, 1T2R and 2R parallel mechanisms," Mechanism and Machine Theory, vol. 61, pp. 184-190, 2013.

[14] X. Kong and C. Gosselin, Type Synthesis of Parallel Mechanisms: Springer London, Limited, 2007.

[15] Z. Huang and Q. C. Li, "Type Synthesis of Symmetrical LowerMobility Parallel Mechanisms Using the Constraint-Synthesis Method," The International Journal of Robotics Research, vol. 22, pp. 59-79, 2003.

[16] Y. Liu, "Symmetry Groups in Robotic Assembly Planning," PhD, Department of Computer and Information Science, University of Massachusetts af Amherst, 1990.

[17] J. M. Selig, Geometric Fundamentals of Robotics: Springer, 1996.

[18] C. Belta and V. Kumar, "Euclidean metrics for motion generation on SE (3)," Proceedings of the Institution of Mechanical Engineers, Part C: Journal of Mechanical Engineering Science, vol. 216, pp. 47-60, 2002.

[19] J. M. Hervé, "Note about the 3-UPU wrist," Mechanism and Machine Theory, vol. 39, pp. 901-904, 2004.

[20] J. M. Hervé, "The Lie group of rigid body displacements, a fundamental tool for mechanism design," Mechanism and Machine Theory, vol. 34, pp. 719-730, 1999.

[21] R. Konkar and M. Cutkosky, "Incremental Kinematic Analysis of Mechanisms," Journal of Mechanical Design, vol. 117, pp. 589-596, 1995.

[22] F. Thomas, "Graphs of kinematic constraints," in Computer-Aided Mechanical Assembly Planning, ed: Springer, 1991, pp. 81-109.

[23] E. Staffetti and F. Thomas, "Analysis of rigid body interactions for compliant motion tasks using the Grassmann-Cayley algebra," in IEEE/RSJ International Conference on Intelligent Robots and Systems, 2000, pp. 2325-2332 vol.3.

[24] Robotiq. 2-Finger Adaptive Robot Gripper - 200. Available: http://robotiq.com/en/products/electric-gripper

[25] L. U. Odhner, R. R. Ma, and A. M. Dollar, "Open-Loop Precision Grasping With Underactuated Hands Inspired by a Human Manipulation Strategy," IEEE Transactions on Automation Science and Engineering, vol. 10, pp. 625-633, 2013.

[26] K. H. Hunt, Kinematic geometry of mechanisms: Clarendon ; Oxford University Press, 1978.

[27] N. Ulrich, R. Paul, and R. Bajcsy, "A medium-complexity compliant end effector," in IEEE International Conference on Robotics and Automation, 1988, pp. 434-436 vol.1.

[28] J. D. Crisman, C. Kanojia, and I. Zeid, "Graspar: a flexible, easily controllable robotic hand," IEEE Robotics \& Automation Magazine, vol. 3, pp. 32-38, 1996.

[29] Lacquey. Fetch Hand. Available: http://www.lacquey.nl/fetch

[30] Robotiq. 3-Finger Adaptive Robot Gripper. Available: http://robotiq.com/en/products/industrial-robot-hand

[31] L. Qinchuan, H. Zhen, and J. M. Herve, "Type synthesis of 3R2T 5DOF parallel mechanisms using the Lie group of displacements," IEEE Transactions on Robotics and Automation, vol. 20, pp. 173-180, 2004.

[32] P. Fanghella and C. Galletti, "Particular or general methods in robot kinematics?: Both particular and general," Mechanism and Machine Theory, vol. 24, pp. 383-394, 1989.

[33] Schunk. SDH Servo-electric 3-Finger Gripping Hand. Available: http://mobile.schunk-microsite.com/en/produkte/produkte.html

[34] Barrett. The BarrettHand, A Multi-fingered Programmable Grasper. Available: http://www.barrett.com/robot/products.htm 